\title{
The Short Wavelength Spectrometer for ISO
}

Th. de Graauw, D. Beintema, W. Luinge, G. Ploeger, K. Wildeman, J. Wijnbergen.

Laboratory for Space Research Groningen, Groningen, The Netherlands.

S. Drapatz, L. Haser, F. Melzner, J. Stőcker.

Max Planck Institut für Extraterrestrische Physik, Garching, F.R.G.

K. van der Hucht, Th. Kamperman, C. van Dijkhuizen.

Laboratory for Space Research Utrecht, Utrecht, The Netherlands.

H. van Agthoven, H. Visser, C. Smorenburg.

Technisch Physische Dienst, Delft, The Netherlands.

\section{Abstract}

The Short Wavelength Spectrometer (SWS) being developed for the Infrared Space Observatory (ISO) is described. The instrument is a pair of grating spectrometers, used in low orders, that will provide resolving powers of about 1000 from $3 \mu \mathrm{m}$ to $45 \mu \mathrm{m}$. In combination with Fabry Perot etalons, the resolving power will be as high as 30,000 over the 15 to $30 \mu \mathrm{m}$ range.

\section{Introduction}

With the implementation of the Infrared Space Observatory (ISO) in ESA's scientific programme the European astronomy community will be enabled to carry out, at infrared wavelengths, deep and detailed investigations of the universe on all scales ranging from extragalactic systems down to planets and comets, unhindered by atmospheric absorption and emission and by warm optics. At ISO, with a $60 \mathrm{~cm}$ cooled telescope optimized for the wavelength range from a few microns to about $100 \mu \mathrm{m}$, spectroscopy is the prime method to obtain a true understanding of the fundamental physical and chemical processes. We have therefore proposed to construct a medium to high resolution spectrometer. This instrument is to cover the short wavelength side of ISO's operating range: $3 \mu \mathrm{m}$ to $45 \mu \mathrm{m}$.

The wavelength regions shortwards of $25 \mu \mathrm{m}$ have been explored over the past 20 years through atmospheric windows from ground-based observatories which has led to photometric and, to much smaller extent, spectroscopic observations of about 10,000 sources (see Catalog of Infrared Observations, Gezari et al., 1984). With a much higher sensitivity the Infrared Astronomical Satellite (IRAS) has surveyed the complete sky in four bands at $12 \mu \mathrm{m}, 25 \mu \mathrm{m}, 60 \mu \mathrm{m}$ and $100 \mu \mathrm{m}$, resulting in a Catalog with about 250,000 point sources ${ }^{2}$ All these sources have been recorded by the IRAS LOW Resolution Spectrograph (LRS) from 8 to 22 um with a resolving power of about 30. About 5500 sources with sufficient Signal to Noise Ratio are published in the LRS catalog (Beintema et al. this symposium). These two catalogs form the prime database for IR spectroscopic programs to investigate physical and chemical processes in the universe.

\section{Scientific Objectives}

The infrared wavelength region to be covered by the SWS includes many atomic, ionic and molecular lines and diffuse spectral features. Several listings exist. For example, Kurucz and Peytreman ${ }^{5}$ listed 20541 spectral lines of all elements between $3 \mu \mathrm{m}$ and $10 \mu \mathrm{m}$, i.e. about 3000 lines per stretch of $1 \mu \mathrm{m}$.

These spectral lines and features provide us with unique and excellent tools for studies of the physical and chemical processes in the universe, particularly of regions optically hidden by interstellar dust. The SWS resolving power allows to probe kinematic processes in a variety of objects ranging from nuclei of galaxies to planetary atmospheres. With the SWS sensitivity, detailed line studies of extragalactic objects out to distances of the Virgo cluster can be carried out. An extensive overview of the broad range of important areas in astrophysics that can be investigated with the SWS, is given in the report of the ISO Science Workshop at Alpbach. A summary of these areas is given in figure 1, where the scientific subjects are divided into five main classes: Solar Systems, Interstellar Matter, Pre-Main Sequence Stars, Post-Main Sequence Stars and Galaxies. The wide variety of astronomical problems that can be studied with the SWS qualifies the spectrometer as an observatory instrument. 


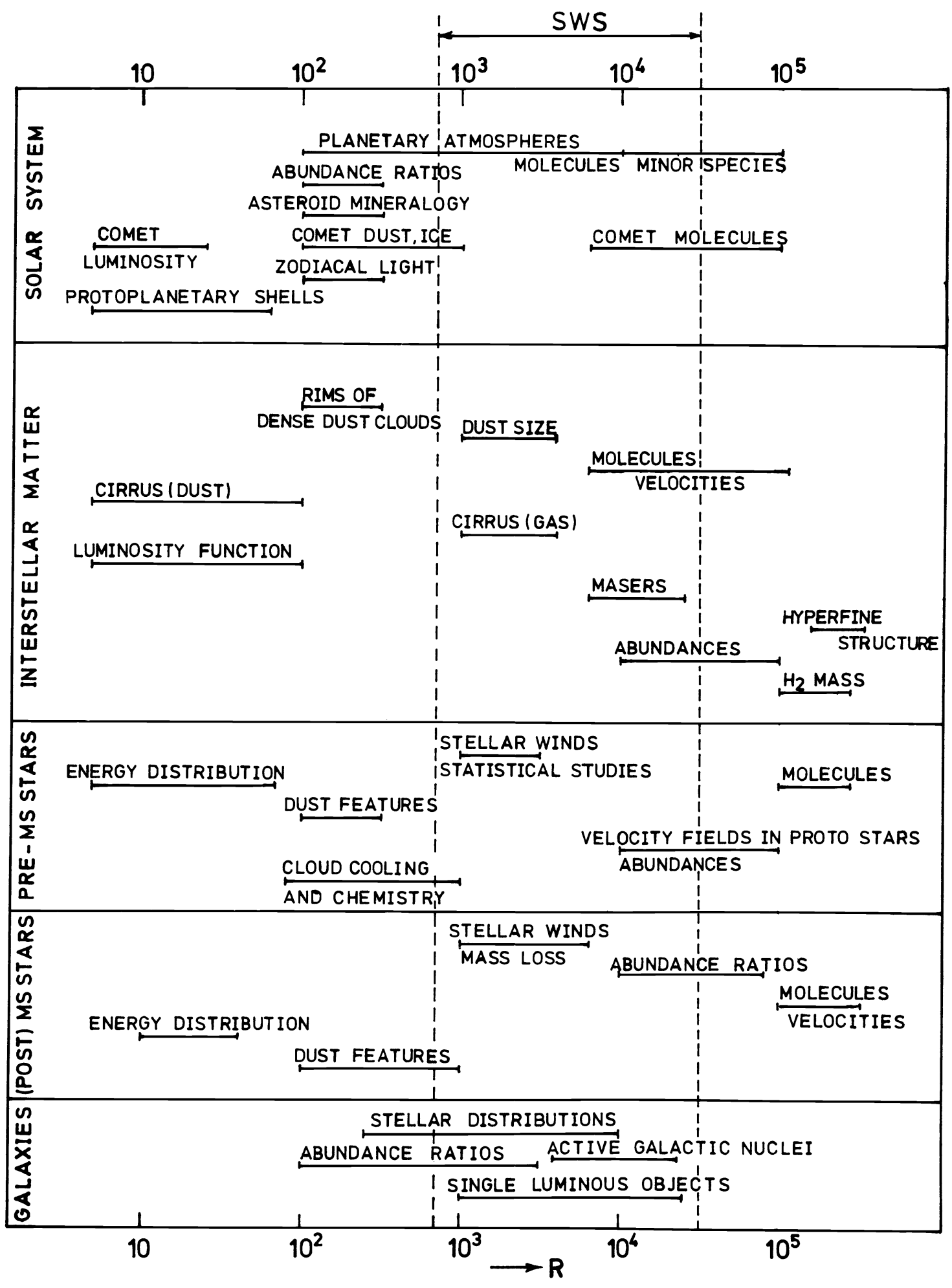

Figure 1. Important areas in astrophysics to be investigated with ISO spectroscopy. The range of resolving power of the SWS is indicated. 
Instrument Description

Introduction

The spectrometer covers a wavelength range from $3 \mu \mathrm{m}$ to $45 \mu \mathrm{m}$ with resolving powers ranging from 1000 to 30,000. The instrument consists basically of two grating spectrometers, used in the first, second and third order. The two spectrometers use the same mechanism for wavelength scanning. They provide a resolving power of about 1000. Two Fabry-Perot interferometers, with the grating spectrometers as predispersers to suppress unwanted F-P orders, cover the wavelength range from $15 \mu \mathrm{m}$ to $30 \mu \mathrm{m}$ with a resolving power of about 30,000 .

The instrument is to fit a quarter of the volume available for instruments, which is a cylinder with $600 \mathrm{~mm}$ diameter and $250 \mathrm{~mm}$ height. Besides the limited dimensions of this sector, the restricted mass (7.5kg) and the small number of available wires going to the cryogenic instrument chamber (60 pairs) the optical design has to be compatible with the limited pointing accuracy of ISO, which does not allow diffractionlimited operation at wavelengths shorter than $20 \mu \mathrm{m}$. Another important constraint for the design is to keep the instrument as simple as possible and to minimize the number of critical failure points e.g. mechanisms.

\section{Optical Concept}

The two grating sections share three entrance apertures located near the focal plane of the telescope which has an unvignetted field of view of 3 arc min. These three entrance apertures are adjacent and situated along the main dispersion direction. Only one of the apertures is used at a time with the help of a shutter that covers the two other apertures.

Directly after these apertures there are reflection filters which reflect the radiation above a certain wavelength. The radiation at shorter wavelengths is transmitted and directed to the short wavelength grating section after having passed a multilayer filter.

The long wavelength radiation is directed to the long wavelength grating section via another reflection filter, in orderto obtain sufficient suppression of the short wavelengths. See figures $2 a$ and $2 b$. In these figures we show the optical layout and the division of the wavelength range ( $3-45 \mu \mathrm{m})$ for the two gratings and the subdivision for the three reflection filters and the three grating orders.

Directly after the reflection filter is the location of the entrance slits, which is in the actual focal plane of the telescope. The width of the slits is 14 or 20 arc seconds. The height of the slits imaged onto the detector is 30 and 40 arc sec respectively. The 14 arc sec width of the entrance slits is mainly determined by the pointing of the telescope. The specified performance for jitter ( 4 arc sec half cone angle) and pointing drift (10 arc sec half cone angle per tour) does not allow to obtain diffraction limited resolving powers at wavelengths below $20 \mu \mathrm{m}$. Directly after these aperture slits two flat mirrors bring the beams to the appropriate collimator and grating section. These two sections are identical except for the grating. The mirrors $M_{1}$ and $M_{2}$ make a collimated beam with an elliptical cross section. These diameters are 20 and 70 $\mathrm{mm}$.

Mirror $M_{1}$ is a saddle shaped mirror and together with the cylindrical mirror $M_{2}$ the image quality in the dispersion direction can be optimized. The collimated beam is reflected by the scanner mirror $M_{3}$ onto the grating. The scanning angle of $M_{3}$ is $\pm 6.3^{\circ}$. The dispersed beam is directed by $M_{3}$ again via $M_{2}$ and $M_{1}$ to mirrors $M_{4}$, which are located just after the spectral image plane. These flat mirrors are adjacent to each other in the dispersion (scanning) direction and direct the beam to the reimaging optics of the spectrum (mirror $M_{8}$ and $M_{9}$ ) or (in case of grating 1) to the collimator, mirrors $M_{5}$ and $M_{6}$, of one of the Fabry-Perot etalon pairs. $M$ focusses the beam of the $F-P$ onto a separate detector. The reimaging is necessary to suppress straylight and to enable the use of the smallest possible detector dimensions. The use of the various detector types as a function of wavelength is given in figure 2.

The option is being considered of extending the wavelength range down to $2.2 \mu \mathrm{m}$, employing another set of detectors with a special band pass filters.

Near the entrance aperture there is the location of two wavelength calibration sources. One for the Fabry-Perot which is mainly used for calibrating the etalon displacers. It consists of a radiation source and a fixed Fabry-Perot with a finesse of about 60. The second wavelength calibrator uses sharp spectral features of a plastic material in front of a radiator at 700K. The Fabry-Perot etalons are made of meshes. Mesh material, now under study, will allow to reach a finesse of about 40 which is sufficient to obtain a resolution of 30,000 with a grating resolution of 750 . An internal radiation source that bypasses the spectrometers and illuminates all detectors simultaneously will facilitate the responsivity calibration of the detectors. A shutter at the entrance of the spectrometer can be used for zero checks. With the aid of a quadrant infrared detector block near the telescope focal plane, the pointing of the telescope can be optimized at the start of an observation and checked during operations. 

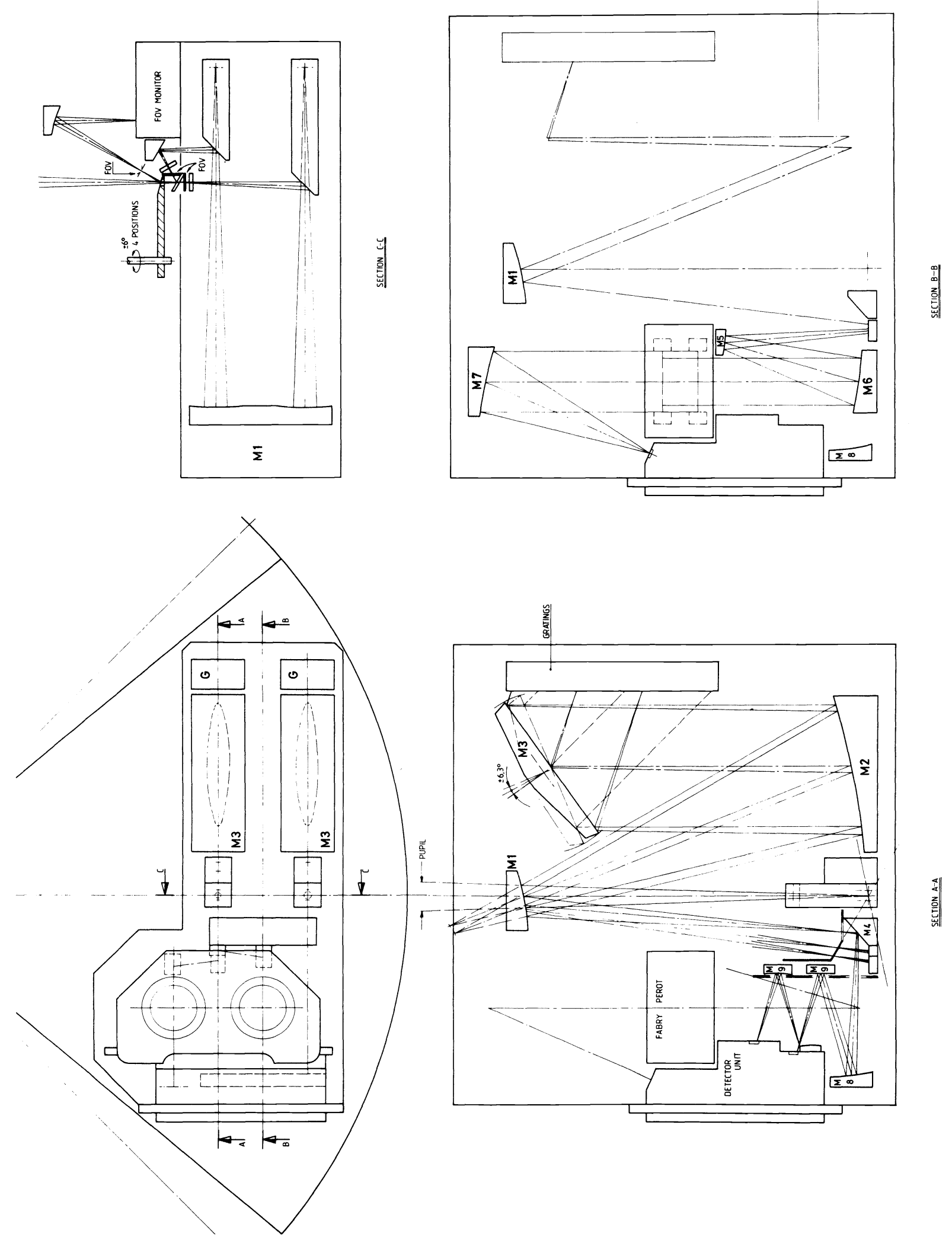

Figure 2a. Schematic lay-out of the Short Wavelength Spectrometer. 


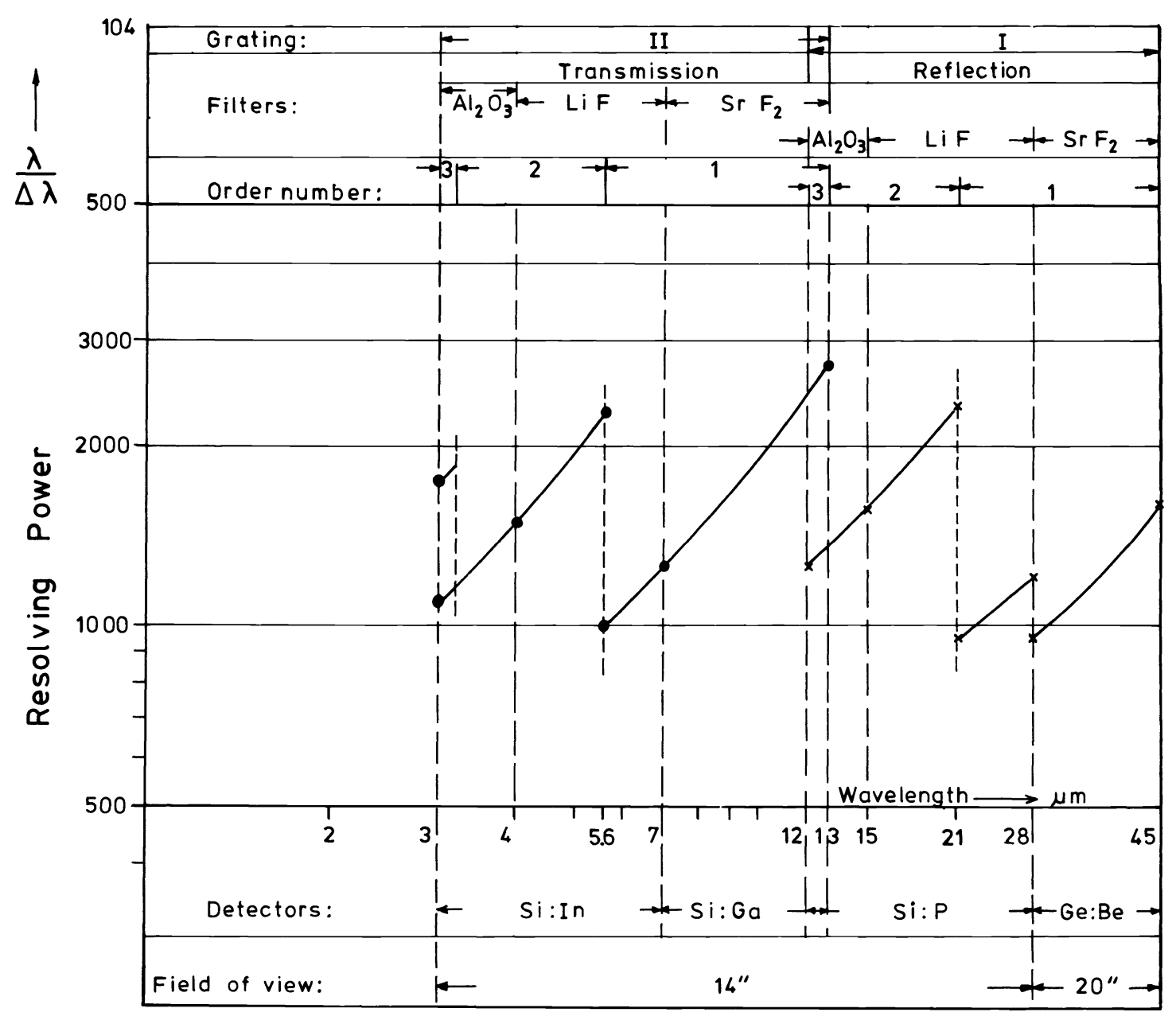

Figure 2b. The theoretical resolving power (no optical aberrations included) of the ISO-SWS is given as a function of wavelength, together with the grating, filter and detector arrangement.

\begin{tabular}{|l|l|l|l|l|}
\hline \multicolumn{4}{|c|}{ Table 1. Overview of the detector types and wavelength range } \\
\hline wavelength range & $3-7.4 \mu \mathrm{m}$ & $7-13 \mu \mathrm{m}$ & $12-28 \mu \mathrm{m}$ & $28-45 \mu \mathrm{m}$ \\
detector material & Si:In & Si:Ga & Si:P & Ge: Be \\
array configuration & monolithic & monolithic & discrete/monolithic & discrete \\
peak response, $\mu \mathrm{m}$ & 5 & 15 & 26.5 & 46 \\
$50 \%$ response, $\mu \mathrm{m}$ & 7.4 & 17.8 & 28.5 & 51 \\
responsivity & $40 \mathrm{~A} / \mathrm{W}$ & $2 \mathrm{~A} / \mathrm{W}$ & $5 \mathrm{~A} / \mathrm{W}$ & $4 \mathrm{~A} / \mathrm{W}$ \\
supplier & AEG & AEG & Battelle & Battelle \\
number of detectors & 12 & 10 & 10 & 8 \\
\hline
\end{tabular}




\section{Detectors and Read-out Electronics}

The spectrometer asks for the application of detector arrays, oriented along the dispersion direction, in order to cover as many spectral elements as possible. Presently the performance of monolithic arrays is severely limited by excessive read-out noise and further developments in multiplexing circuits are still necessary to provide a competitive low-noise solution as compared to single-channel circuits. We have therefore taken the approach to aim at applying detector array technology wherever possible in combination with single-channel read-out.

An important constraint in the selection of the design of the detector and read-out circuitry is the sensitivity to high-energy particle radiation. In general discrete detectors are larger, and thus more susceptible to particle hits. However, discrete detectors can be made as small as $150 \mu \mathrm{m} \times 150 \mu \mathrm{m}$, monolithic detector array elements may have dimensions of $50 \mu \mathrm{m} \times 50 \mu \mathrm{m}$. Table 1 gives an overview of the spectral ranges and detector types used in the SWS.

The restricted number of wires going into the cryostat limits the number of detectors and is an important consideration for the final selecting of the read-out circuits.

The baseline for the detector read-out is to have each detector connected with an integrating amplifier. The configuration is similar to that described by Low. ${ }^{3}$ Basically only a single heated JFET is needed, requiring a minimum amount of wires. The read-out will be non-destructive. Only after reaching the range limits, the integration circuit will be reset by a MOSFET switch. Measurements in our laboratory have shown that the Noise Equivalent Current can be as low as $10^{-17} \mathrm{~A} / \sqrt{\mathrm{Hz}}$ at frequencies below $10 \mathrm{~Hz}$. With the responsivities from table 1, NEP's of $2 \times 10^{-18} \mathrm{~W} / \sqrt{ } \mathrm{Hz}$ can be obtained, in agreement with reports by Low. Ultimately the sensitivity may be limited by other causes, such as electrical interference or the high energy radiation environment in space.

We therefore quote both an NEP of $2 \times 10^{-18} \mathrm{~W} / \mathrm{VHz}$ as a design goal and a 5 times higher value as a conservative sensitivity estimate. With these values we calculated the limiting flux densities of figure 3 , assuming an optical effiency of $10 \%$ for the grating spectrometers and $5 \%$ for the F-P's. With the F-P's we assumed that 8 integrations at different wavelengths are needed (in the grating mode the number of detectors in each band is sufficient to measure a spectral line and its adjacent continuum simultaneously).

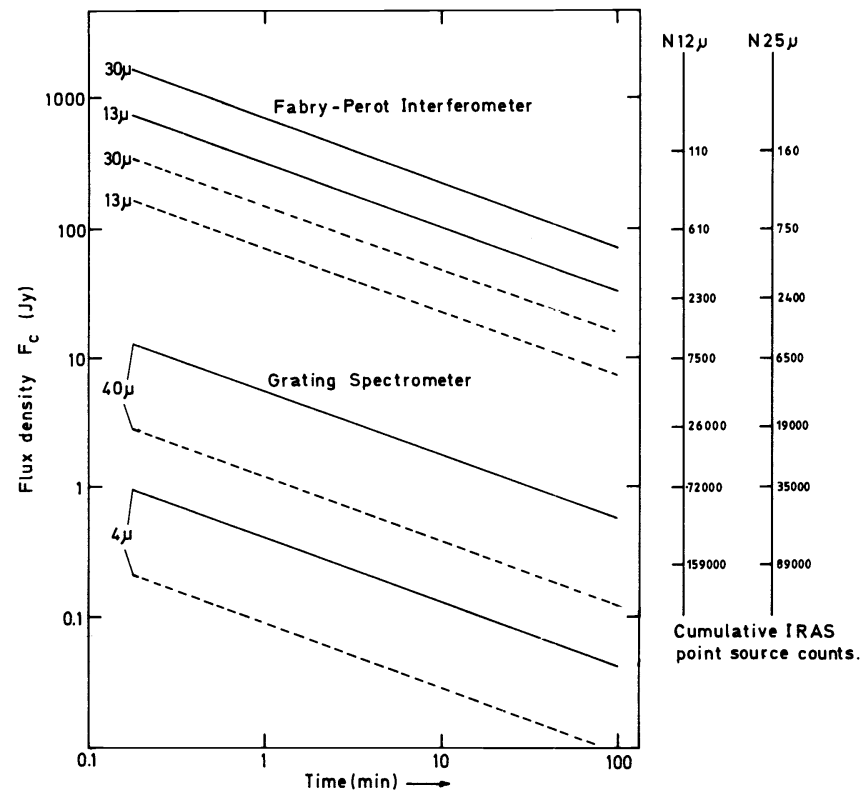

Figure 3. Limiting flux densities $(S N R=10)$ for spectral-line obseryations as a function $8 \mathrm{f}$ observing time, for two detector NEP's : $10^{-17} \mathrm{~W} / \sqrt{\mathrm{Hz}}$ and $2 \times 10^{-18}$ Watt/ $\sqrt{\mathrm{Hz}}$ (dotted line). Cumulative IRAS point source counts are indicated on the right. 


\section{Cryomechanisms}

The optical design requires rotations of the scanning mirror M3 over a 13-degree range. Deflection angles no larger than 6.5 degrees allow the application of flexural pivots in combination with a linear motor. A mechanism based on this principle has been developed and successfully tested for the ISO phase A Michelson spectrometer programme.

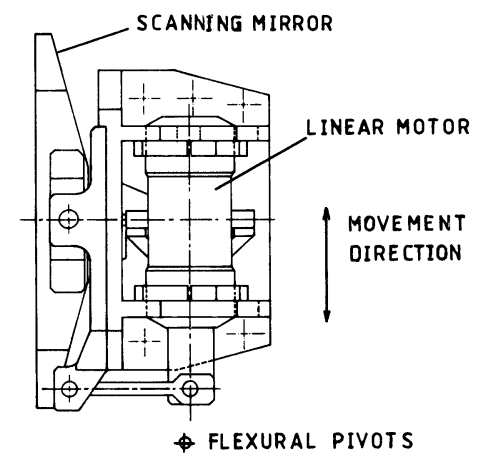

Figure 4. The scanner mechanism.

There are important advantages to this type of mechanism, particularly in a cryogenic environment. From the technical point of view it is a reliable concept because of the absence of bearings and reduction gears, with their associated risks of wear and malfunction. The power dissipation and the drive current are very small ( $<5 \mathrm{~mW},<5 \mathrm{~mA}$ ), which minimizes also the problem of power dissipation in the cryostat cabling.

The mirror drive mechanism shown in figure 4 is presently being tested. With four pivots an approximately linear motion is obtained for the motor. Another design with only two pivots will soon be tested as well. The shutter mechanism will be of the same type.

\section{Conclusion}

In conclusion, we have described the short-wavelength spectrometer for ISO. The present optical concept and several other important instrumental elements e.g. mechanisms, detector read-out, F-P meshes, are under development. The predicted instrument performance will be sufficient to allow spectral observations of the weakest sources detected by IRAS, with resolving powers around 1000 .

\section{References}

1. Gezari, D.Y., Schmitz, M., and Mead, J.M., Catalog of Infrared Observations, NASA Ref. Publ. no.1118, 1984.

2. Joint IRAS Working Group, IRAS), Catalogs and Atlases, Explanatory Supplement, 1984.

3. Low, F. , Appl. Optics 23, 1308, 1984 .

4. ISO Science Workshop, Alpbach Austria, 1984. C. Cesarsky and Th. de Graauw editors.

5. Kurucz, R.L., Peytreman, E., A table of Semi-empirical gf Values, Part 3, SAO-SR 362 $362,1975$. 